\title{
Revisiting the Stark Width and Shift of He II P $\alpha$
}

\author{
Christine Stollberg ${ }^{1, *(1)}$, Evgeny Stambulchik ${ }^{1}$ (1), Bin Duan 2 (1), Marco A. Gigosos ${ }^{3}$ (1), \\ Diego González Herrero ${ }^{3}$, Carlos A. Iglesias 4 (i) and Caroline Mossé 5 (i) \\ 1 Faculty of Physics, Weizmann Institute of Science, Rehovot 7610001, Israel; \\ Evgeny.Stambulchik@weizmann.ac.il \\ 2 Institute of Applied Physics and Computational Mathematics, Beijing 100088, China; duan_bin@iapcm.ac.cn \\ 3 Departamento de Óptica, Universidad de Valladolid, 47071 Valladolid, Spain; \\ gigosos@coyanza.opt.cie.uva.es (M.A.G.); diego.gonzalez@kuleuven.be (D.G.H.) \\ 4 Lawrence Livermore National Laboratories, P.O. Box 808, Livermore, CA 94550, USA; iglesias1@llnl.gov \\ 5 Aix Marseille Université, CNRS, PIIM UMR 7345, 13397 Marseille, France; caroline.mosse@univ-amu.fr \\ * Correspondence: christine.stollberg@weizmann.ac.il; Tel.: +972-8-934-6923
}

Received: 1 March 2018; Accepted: 18 April 2018; Published: 24 April 2018

\begin{abstract}
We report experimental determination of plasma-induced Stark widths and shifts of the $\mathrm{He}$ II $\mathrm{P} \alpha$ line and a comparison of the results with calculations performed by several computational approaches. The measurements were carried out in a small compressing plasma channel device, reaching electron densities in excess of $10^{18} \mathrm{~cm}^{-3}$ and temperatures of a few $\mathrm{eV}$. The experimental data are in a good agreement with some previously published studies. However, the measured relation between the Stark shift and width could not be reproduced by either of the codes, and this disagreement is not yet resolved. This suggests the existence of an additional effect that is not accounted for in the present models and leads to a larger than expected Stark shift of the He II P $\alpha$ line.
\end{abstract}

Keywords: Stark width and shift; helium Paschen- $\alpha$; lineshape code comparison; plasma density diagnostics

\section{Introduction}

Lineshape analysis is a powerful tool for non-intrusive diagnostics of plasma density. Both the width and the shift of a lineshape can be used for diagnostics purposes. In general, the shift is less frequently used than the width, since it is usually smaller by magnitude and its relative theoretical uncertainties are higher [1]. However, the Stark shift has a unique advantage over the width: it is unaffected by the opacity and Doppler broadening (in the absence of macroscopic plasma motion). On the other hand, the measurement of the Stark shift is a delicate procedure that requires accurate wavelength calibration. Ultimately, both width and shift measurements should provide consistent diagnostic results.

The singly ionized, hydrogen-like helium atom is one of the simplest atomic systems that is very useful for plasma diagnostics. A number of studies have been devoted to determining the Stark width and shift of He II P $\alpha$ ( $n=4 \rightarrow n=3$ transition) and comparing them to available analytical models [2-7]. Given the challenges of measuring the shift precisely, it is perhaps not surprising that the experimental data on He II P $\alpha$ reported in the literature have a noticeable spread. Indeed surprising, however, is a very good agreement between some data, i.e., [2,5-7] or [3,4] but a strong disagreement between those data sets.

The present study includes new experimental data, obtained in a recent experiment performed on a small-scale self-compressing plasma channel. The time- and space-integrated character of the present data enables a precise analysis of Stark shift $d$ and width $w$ independent of an absolute wavelength calibration. Such common sources of error as a reference wavelength, uncertainties in the 
dispersion, and spectrometer aberrations can be excluded. The obtained data agree very well with those of Büscher et al. [6]. However, the measured relation between the Stark shift and Stark width could not be reproduced by the lineshape simulation code SimU [8]. Therefore, the experimental data were included as an "experimental" case in the 3rd Spectral Line Shapes in Plasmas code comparison workshop [9].

During the workshop, the results of several computational codes were compared. The codes were tested on a set of pre-defined plasma parameters that are typical for the experimental conditions. However, none of the codes was able to reproduce the experimentally observed relation between Stark width and shift of He II P $\alpha$. Therefore, the riddle of an anomalously large Stark shift of the He II P $\alpha$ transition remains unresolved.

\section{Experiment}

\subsection{Experimental Setup}

The experimental data were obtained by spectroscopy of a small-scale self-compressing plasma channel. The plasma generation system is shown in Figure 1. A hollow de-Laval nozzle with an outer diameter of $2.4 \mathrm{~mm}$ was used to inject a helium column into the 4-mm anode-cathode gap. To ensure a uniform discharge, the gas was pre-ionized by 4 carbon-brush electron guns. Subsequently, a 24-kA current pulse with a rise time of $260 \mathrm{~ns}$ was driven through the gas load. The discharge ionized the gas and generated an azimuthal magnetic field that accelerated the plasma radially inward. The plasma shell imploded with decreasing radius and increasing electron density, until it eventually stagnated on the axis. The plasma life time was $\approx 300 \mathrm{~ns}$. Typical plasma parameters were $n_{e}<10^{19} \mathrm{~cm}^{-3}$ and $3 \mathrm{eV}<T_{e}<7 \mathrm{eV}$, as obtained by time-resolved spectroscopy (not presented here).

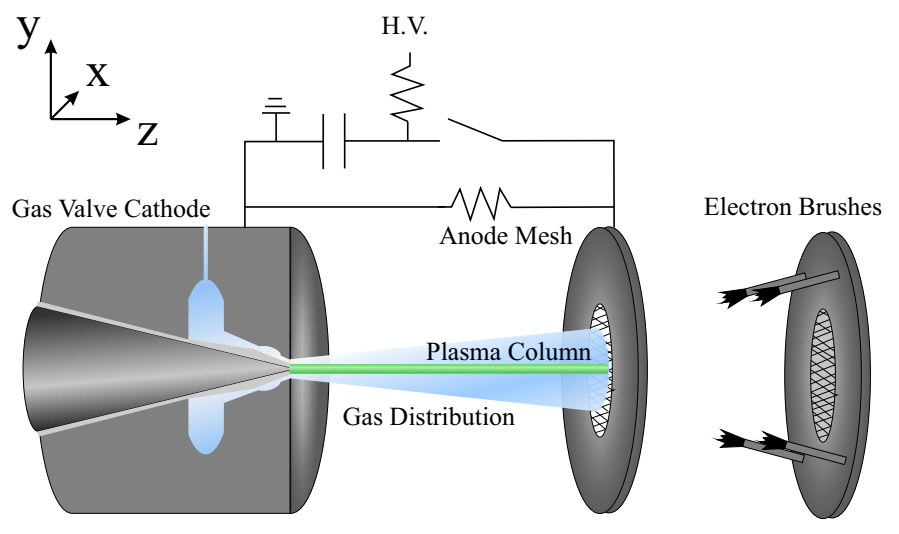

Figure 1. Plasma generation system.

The plasma was diagnosed from the radial direction with a high-resolution spectroscopic setup. An Andor Shamrock 303i imaging spectrometer, equipped with a 2400 lines/mm grating was coupled with an Apogee Alta U900 CCD with an exposure time of $0.3 \mathrm{~s}$. A spatial and spectral resolution of $200 \mu \mathrm{m}$ and $1 \AA$, respectively, and a dispersion of $0.047 \AA /$ px were achieved. These parameters were confirmed by measuring the spectrum of a mercury DC low-pressure calibration lamp.

\subsection{Experimental Data}

The diagnostic setup yielded a time- and space-integrated observation (along the radial line of sight) of the compressing plasma channel. The recorded spectral image is shown in Figure 2a. In Figure $2 b$, the plasma channel is sketched at different times of compression with decreasing radius and increasing electron density. Figure $2 c$ contains the intensity profiles obtained at different times by spatial integration over the line of sight along $x$. The additional integration in time resulted in an accumulated intensity profile. At large $y$ positions, the image contained only information from large 
radii, at which the plasma resided at early stages of the implosion. In the central part of the image $(y=0)$, light from all stages of the compression was collected. It is worth noting that the spectral image, Figure 2a, exhibits strong aberrations caused by the spectrometer imaging optics. This can be corrected by recording the spectrum of a calibration lamp. However, our analysis method used the recorded data as illustrated in Figure 2a with no additional corrections.

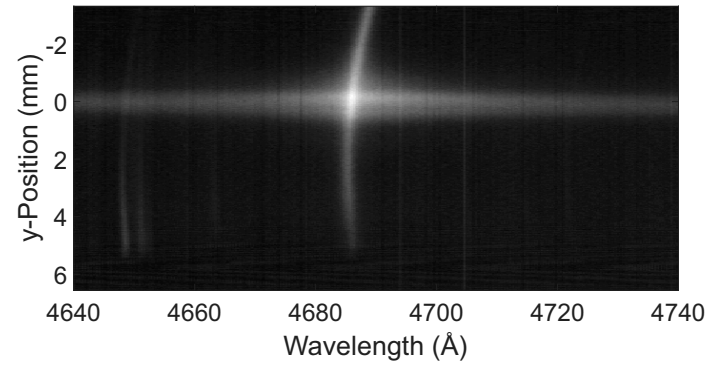

(a)

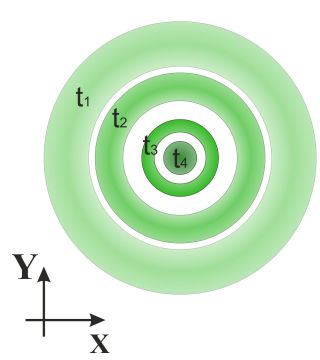

(b)

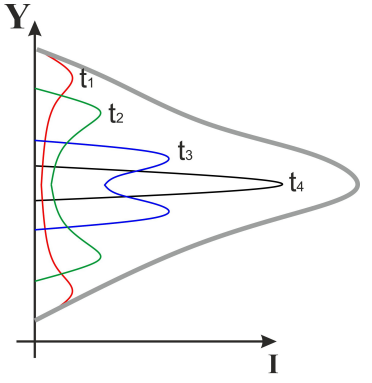

(c)

Figure 2. (a) Spectral image of the time and space integrated He II P $\alpha$ line, (b) sketch of different stages of the compressing plasma channel, and (c) spatially (along $x$ ) integrated intensity profiles originating at different times from the compressing plasma channel and the time-integrated profile (the thick gray line).

In Figure 3a, we show spectral lineouts taken from Figure $2 a$ at different $y$ positions. At large $y$ values, the low-density plasma from the early stages of compression creates a narrow line profile almost at the original wavelength. In the following, we will refer to the low-density plasma as the "reference plasma," which creates a "reference line." Towards lower y positions, radiation from the compressed, denser plasma was collected. This radiation was prone to the Stark effect, resulting in broadening and shifts. Due to the spatial and temporal integration of the detector, as demonstrated in Figure $2 b, c$, the signals of the reference plasma and the high-density plasma could be observed simultaneously at the same $y$ positions. Although the low-density plasma had an intrinsically lower emission intensity, the slower rate of acceleration at the beginning of the plasma compression led to a longer exposure time for the reference plasma. Therefore, the reference line was still strong in comparison to the high-density plasma profile. Thus, at each $y$ position, we measured the line profile of the high density plasma together with the "reference line" of the reference plasma. The superposition of the unshifted, narrow reference line and the broadened, shifted high-density-plasma profile caused a broad and asymmetric spectral lineshape, as seen in Figure 3a,c. We emphasize again the fact that we are not comparing the shifted line profile from the high density plasma at $y \approx 0$ to the unshifted reference line at $y \gg 0$. Instead, the reference plasma produced a reference line over the whole $y$-range of the detector due to the time- and space-integrated character of the measurement. As the reference line and the high-density profile at each $y$ position followed the same optical path, their spectral offset was not affected by spectrometer aberrations but resulted solely from the Stark effect.

An example of fitting of a spectral line profile at two different $y$ positions is shown in Figure $3 b, c$. The profile in Figure $3 b$, recorded at the outer edge of the image, is fitted with a single Voigt profile. The residue shows that there is no recognizable asymmetry in the He II P $\alpha$ line at low densities. In agreement with the literature [7] and our simulations, we assumed that there was no significant asymmetry at higher densities. Moreover, the absence of impurity lines is indicated by the residual data. The Lorentzian part of the Voigt fitting profile, which corresponds to the Stark broadening, is $1.8 \pm 0.4 \AA$, while the Gaussian part is fixed to $1.4 \AA$ and accounts for the instrumental, Doppler, and Zeeman broadenings.

The lineshape in Figure $3 c$ bears a strong asymmetry and cannot be fitted with a single Voigt profile. A two-Voigt fit, however, yields acceptable results, as seen from the residue. The offset between the central wavelengths of the reference line and the shifted line constitutes $3.1 \AA\left(-14.1 \mathrm{~cm}^{-1}\right)$. 
The full width at half-maximum (FWHM) of the fitted lines are $2.3 \AA\left(10.5 \mathrm{~cm}^{-1}\right)$ and $37.4 \AA\left(169 \mathrm{~cm}^{-1}\right)$, respectively. We can, therefore, directly relate the width of the high-density line to the Stark width of $37.4 \AA$ and relate the offset between the two lines to the Stark shift of $3.1 \AA$. Considering the $d-w$ relation makes the analysis independent of uncertainties in the dispersion. In principle, the relation could also be given in pixels of the detector. However, for the sake of the comparison of our results to the literature, we present here our data in units of $\AA$ or $\mathrm{cm}^{-1}$. Note that, at the relevant wavelength, $1 \AA$ corresponds to $\approx 4.55 \mathrm{~cm}^{-1}$.

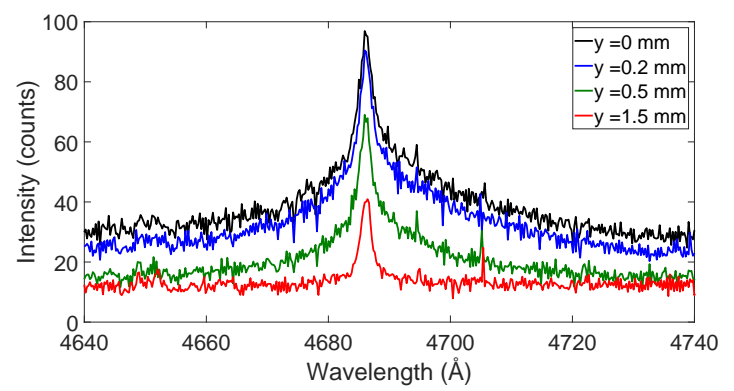

(a)

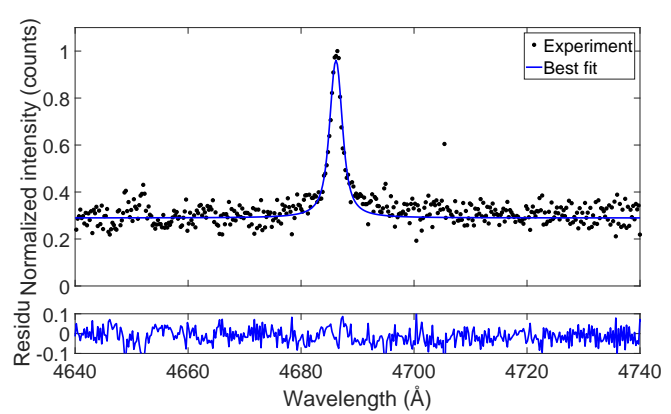

(b)

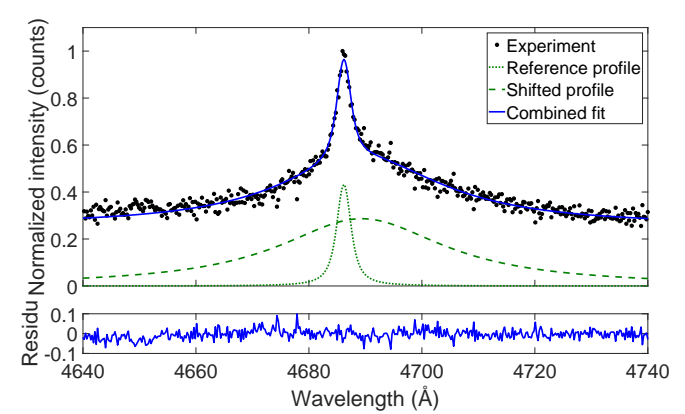

(c)

Figure 3. (a) Spectral lineouts at different $y$ positions; (b) spectral lineout at $y=1.5 \mathrm{~mm}$ and (c) at $y=0$ with their best fits.

The Stark shifts and widths obtained at different $y$ positions are summarized in Table 1 and their relation is shown in Figure 4. The error bars provided in Figure 4 result mainly from the confidence intervals of the best fit of the experimental profiles. An additional uncertainty is due to the fact that the measured reference line does not represent the zero electron density, but rather a relatively low density (FWHM $\lesssim 3 \AA$ ). The error in the measured shift can be estimated to be below $0.2 \AA$ and is strictly towards larger shifts. Further sources of error have only a minor influence: The opacity was calculated to be below 0.1 for a large range of parameters and 0.3 at most for $n_{e}=10^{19} \mathrm{~cm}^{-3}$ at $T_{e}=10 \mathrm{eV}$. The broadening of the He II P $\alpha$ profile due to the opacity is, therefore, below $10 \%$. The influence of a potential macroscopic Doppler effect due to the motion of the entire plasma column can be estimated to be well below $0.1 \AA$.

In Figure 4, the best fits to selected data from the literature are shown. The experimental data obtained in the present study agree well with the data from Büscher et al. [6] and Gawron et al. [5]. However, a large deviation from the data of Kobilarov et al. [4] was observed. The data of Pittman and Fleurier [3] and Fleurier and Gall [2] are in a significantly lower density range, so no direct comparison to the present data can be drawn. While there are more data on the Stark shift of He II P $\alpha$ available in the literature (see references in [6]), this small selection illustrates the inconsistency of the experimental Stark shift data in relatively similar experiments. In all the experiments compared here, no intrinsic differences between the two distinct data sets [2,5-7] and [3,4] in terms of plasma source, working gas, or observation direction could be found. The experimental details are summarized in Table 2. 
Successful modeling of data by either a suitable theory or simulation might reveal a crucial difference in plasma conditions that leads to the different Stark shifts.

Table 1. Summary of the experimental data.

\begin{tabular}{cccc}
\hline $\boldsymbol{y}$ position $(\mathrm{mm})$ & $\boldsymbol{w}_{\text {ref }}(\AA)$ & $\boldsymbol{w}_{\text {shifted }}(\AA)$ & $\boldsymbol{d}_{\text {shifted }}-\boldsymbol{d}_{\text {ref }}(\AA)$ \\
\hline-1.0 & $2.6 \pm 0.1$ & $20.8 \pm 3.1$ & $1.8 \pm 1.0$ \\
-0.8 & $4.0 \pm 0.5$ & $23.6 \pm 2.8$ & $2.2 \pm 0.6$ \\
-0.5 & $2.9 \pm 0.5$ & $28.7 \pm 2.4$ & $2.9 \pm 0.5$ \\
-0.2 & $3.0 \pm 0.7$ & $38.1 \pm 3.8$ & $3.8 \pm 0.8$ \\
0.0 & $2.6 \pm 0.7$ & $35.9 \pm 5.3$ & $3.2 \pm 1.1$ \\
0.1 & $2.4 \pm 0.6$ & $37.8 \pm 5.5$ & $3.0 \pm 1.1$ \\
0.3 & $2.5 \pm 0.5$ & $35.0 \pm 4.0$ & $3.0 \pm 0.8$ \\
0.5 & $2.3 \pm 0.5$ & $29.0 \pm 4.0$ & $2.7 \pm 0.9$ \\
\hline
\end{tabular}

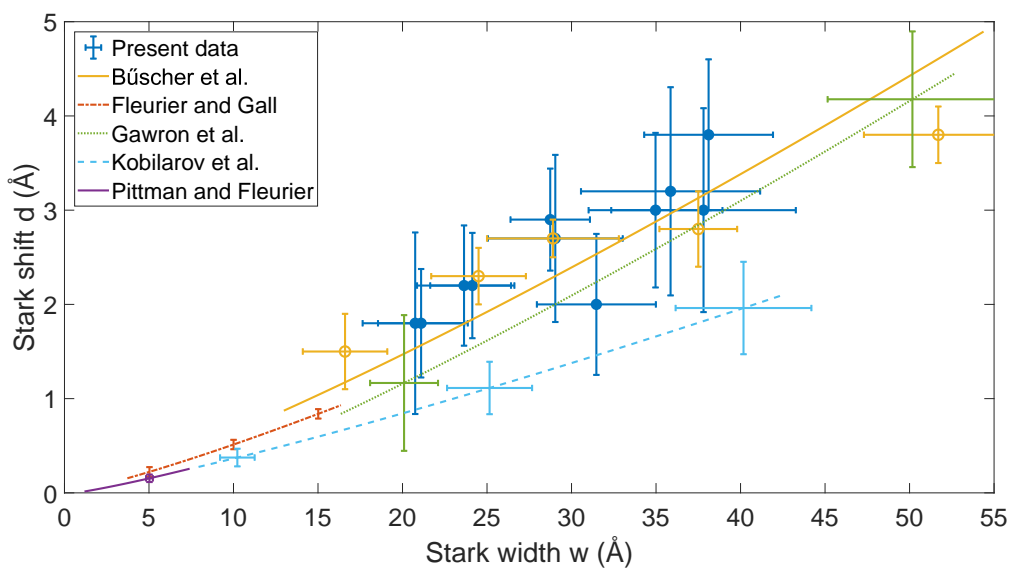

Figure 4. Experimentally obtained width and shift. The results from the present study are compared to different data from the literature.

Table 2. Details on experimental setups from the literature.

\begin{tabular}{cccc}
\hline Reference & Plasma Source & Working Gas & Observation Direction \\
\hline Present data & Gas liner z-pinch & He & Radial \\
Büscher et al. [6] & Gas liner z-pinch & H with He doping & Radial \\
Fleurier and Gall [2] & Capillary z-pinch & He & Axial \\
Gawron et al. [5] & Gas liner z-pinch & H with He doping & N/A \\
\hline Kobilarov et al. [4] & Capillary z-pinch & He & Axial \\
Pittman and Fleurier [3] & Capillary z-pinch & He & Axial \\
\hline
\end{tabular}

\section{Code Comparison}

\subsection{Description of Participating Codes}

Participating codes are summarized in Table 3. Among the seven codes, there are four models and three simulations. (Strictly speaking, the computer simulations are also variants of Stark broadening models [10], but we here use this simplified categorization for brevity.) The quenching property (accounting for the $\Delta n \neq 0$ couplings) is mentioned in particular here, as the quenching is crucial for obtaining the Stark shift of any hydrogen-like transition [11]. The codes are described in the references listed in the table. Furthermore, a comparative description for most of these codes (ER, MELS, PPP, QC-FFM, and SimU) is given elsewhere [12]. Therefore, only MD and ST will be briefly described here. 
Table 3. Summary of the participating codes.

\begin{tabular}{cccc}
\hline Code & Type & Quenching & Reference \\
\hline ER & Simulation & No & {$[13]$} \\
MD & Simulation & No & {$[14]$} \\
MELS & Model & Yes $^{a}$ & {$[15]$} \\
PPP & Model & No & {$[16]$} \\
QC-FFM & Model & No & {$[17]$} \\
SimU & Simulation & Yes $^{a}$ & {$[8]$} \\
ST & Model & Yes & {$[18]$} \\
\hline${ }^{a}$ Calculations performed with and without quenching enabled.
\end{tabular}

MD uses the same approach as ER for solving the Schrödinger equation. Namely, for hydrogen(-like) atomic systems and when the no-quenching approximation is considered, the atom state is described with the Euler-Rodrigues parameters [19]. The principal difference is in simulating the plasma particles, which in MD is done by directly solving a pure Coulomb N-body problem (Debye quasiparticles are not used: the shielding and plasma coupling arise naturally).

ST [18] is a method for the calculation of lineshapes by the "standard theory" [1], which is based on the quasi-static approximation for plasma ions and impact approximation for plasma electrons. In these calculations, the Dirac $R$-matrix methods are used to calculate the electron-impact broadening operator, which is assumed to be a diagonal matrix for simplicity. The method of Tighe and Hooper [20] is chosen to calculate the electric microfield distribution function. The dipole matrix and the required atomic structure data are calculated by the atomic structure package GRASP [21].

The most relevant details of the participating models and simulations are compared in Tables 4 and 5 , respectively. In all calculations presented here, $T_{e}=T_{i}=T$ was assumed.

Table 4. Details of participating models.

\begin{tabular}{ccccc}
\hline Code & Levels & Microfields & Electrons & Ion Dynamics \\
\hline MELS & Detailed & APEX [22] & Relaxation impact [23] & BID [24] \\
PPP & Detailed & APEX & Standard theory impact & FFM [25] \\
QC-FFM & QC [26] & Pfennig and Trefftz [27] & FFM w/ impact corr. [17] & FFM w /impact corr. \\
ST & Detailed & Tighe and Hooper [20] & K-matrix impact & - \\
\hline
\end{tabular}

Table 5. Details of participating simulations. In all three codes, the motion of all plasma particles_electrons and ions-is modeled.

\begin{tabular}{ccc}
\hline Code & Plasma model & Ion Shielding \\
\hline ER & Debye QP's, straight paths & By electrons only \\
MD & True Coulomb MD & N/A \\
SimU & Debye QP's with explicit RPI & By electrons and ions \\
\hline
\end{tabular}

\subsection{Code Results in Model Cases}

In order to directly compare the results of the different codes with each other, a grid of plasma parameters was used: $n_{e}=10^{18} \mathrm{~cm}^{-3}$ and $10^{19} \mathrm{~cm}^{-3}, T=4 \mathrm{eV}$ and $10 \mathrm{eV}$. In addition, two atomic models were suggested for analysis, without and with quenching. Therefore, in total, eight sub-cases were defined. The first four sub-cases without quenching are summarized in Figure 5. 


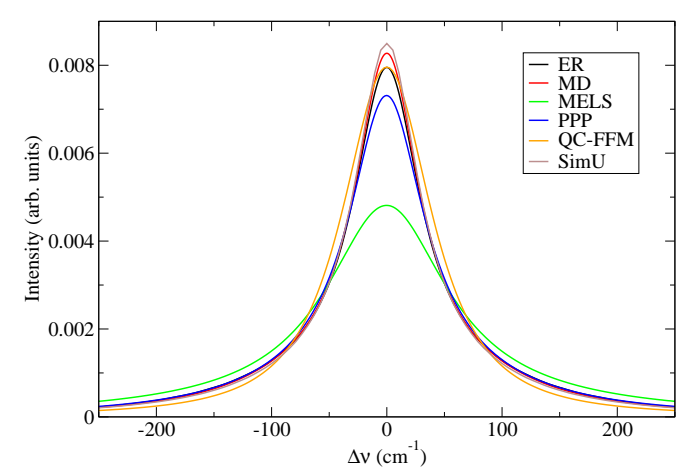

(a)

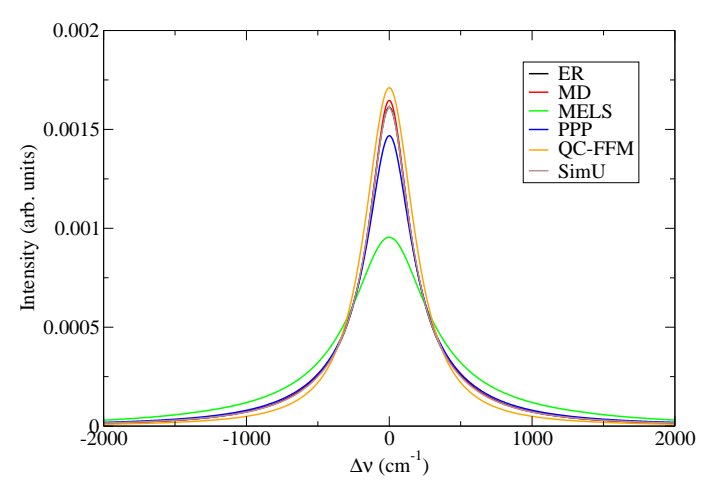

(c)

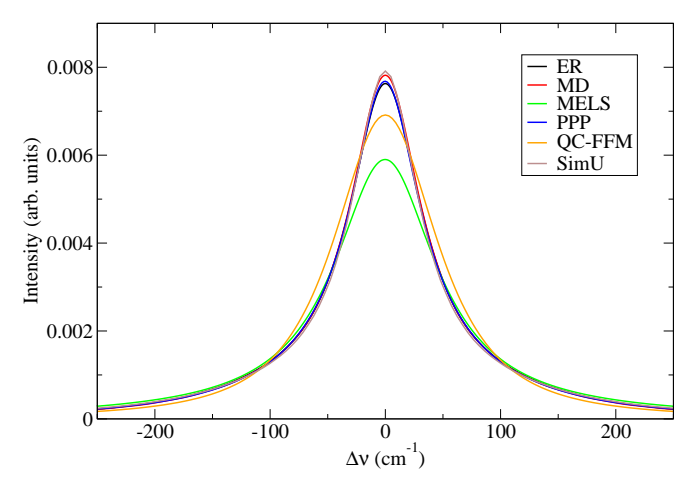

(b)

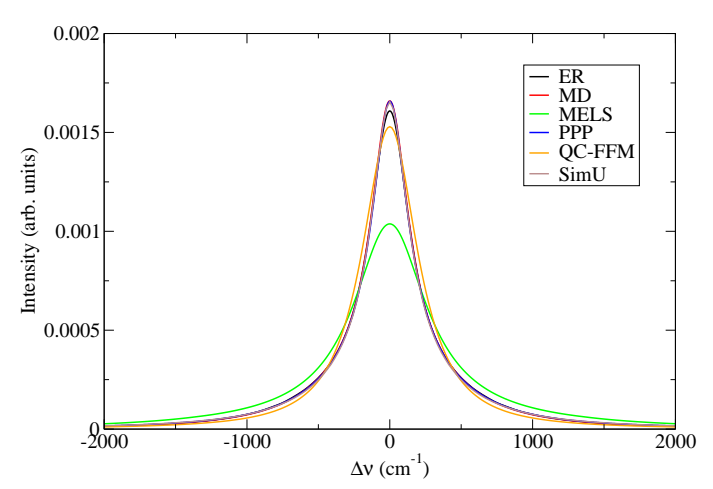

(d)

Figure 5. Sub-cases without quenching. Plasma conditions assumed as follows: (a) $n_{e}=10^{18} \mathrm{~cm}^{-3}$ and $T=4 \mathrm{eV} ;(\mathbf{b}) n_{e}=10^{18} \mathrm{~cm}^{-3}$ and $T=10 \mathrm{eV} ;(\mathbf{c}) n_{e}=10^{19} \mathrm{~cm}^{-3}$ and $T=4 \mathrm{eV} ;(\mathbf{d}) n_{e}=10^{19} \mathrm{~cm}^{-3}$ and $\mathrm{T}=10 \mathrm{eV}$.

The lineshapes obtained by the simulations agree very well in all sub-cases. The main principal difference between these three codes is their approach to modeling the plasma microfields. ER and SimU assume Debye quasiparticles (QPs), while MD implements true Coulomb N-body molecular dynamics and therefore should be the most accurate. ER assumes that perturbers move along straight paths, while SimU takes into account interactions between the radiator and perturbers (but not between perturbers). Furthermore, SimU ions are assumed to be screened by electrons and ions themselves, while ER ions are assumed to be screened only by electrons. It is thus expected that SimU yields smaller linewidths than ER does due to the effective treatment of the perturber-perturber interactions that are either somewhat overestimated (SimU) or underestimated (ER), with the more accurate treatment of MD in between. Indeed, this is what is observed in the results presented here. However, the differences in the results between all the three approaches are rather minor for the modestly coupled plasmas considered here.

The models, on the other hand, exhibit a more significant spread. In all cases, the line widths obtained by MELS are noticeably larger than that of the other codes. This is believed to be due to the O'Brien-Hooper [23] electron width operator used. Such differences have been previously shown [28]: the $\mathrm{O}^{\prime}$ Brien-Hooper formulation for electron impact widths is less reliable for lines involving higher principal quantum numbers [29] since it does not have an $n$-dependent cutoff. The results of QC-FFM have to be regarded cautiously, as the underlying QC model [26] was specifically designed for $\Delta n \gg 1$ transitions. Lines with $\Delta n=1$, such as Paschen- $\alpha$ discussed here, require special treatment of the central component. On the other hand, the relative contribution of the central component in this line is significantly smaller than, e.g., that in = Lyman- $\alpha$ [30]; therefore, the QC-FFM lineshapes are comparable to those of the more complex codes. The density dependence of the Stark width of all codes is approximately $w \propto n_{e}^{2 / 3}$. 
Figure 6 summarizes the calculation results for the participating codes where quenching is available. For MELS, the levels with $n=3-5$ are included, SimU incorporates levels 3-6, and ST uses levels 1-5. Including higher levels in the Hamiltonian affects the lineshape. All participating codes produce slightly asymmetric profiles with an elevated red wing. Due to the small level of the asymmetry, the line widths and centers can be obtained with reasonable accuracy $(\approx 15 \%)$ by fitting them with a Lorentzian profile. Over a wide range of parameters, the ST model exhibits the lowest line width, likely due to the neglect of ion dynamics. Indeed, the best agreement between all three codes is observed at the highest- $n_{e}$ /lowest- $T$ grid point (Figure $6 \mathrm{c}$ ), while the worst agreement is observed for the lowest- $n_{e} /$ highest- $T$ (Figure $6 \mathrm{~b}$ ). Other than that, the leading density dependence remains the same as in the no-quenching case, $w \propto n_{e}^{2 / 3}$.

The quenching also leads to the Stark shift. All profiles show a "red" shift, and the codes that give broader lineshapes also give larger shifts. It should be noted that MELS does not include line shifts due to the electron collisions; only ions contribute to the shift. The results are summarized in Figure 7. For the sake of comparison, the shifts are scaled to the density of $n_{e}=10^{18} \mathrm{~cm}^{-3}$. It can be seen that all calculations demonstrate a slightly weaker than linear dependence on the density. This is in part due to the screening effect, resulting in a decrease of the microfield magnitudes and, hence, a smaller ion-induced shift. Such a positive correlation between the plasma temperature and the shift is indeed observed in the MELS results (recall these are only ion-induced shifts). On the other hand, the electron-induced shift in the impact approximation should decrease with $T$, which is observed in the ST results, whereas the SimU shift is practically independent of $T$, which we attribute to a significant cancellation of the two effects.

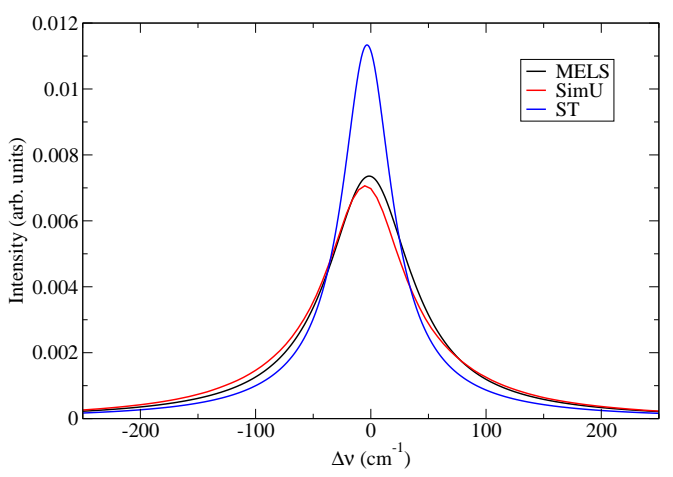

(a)

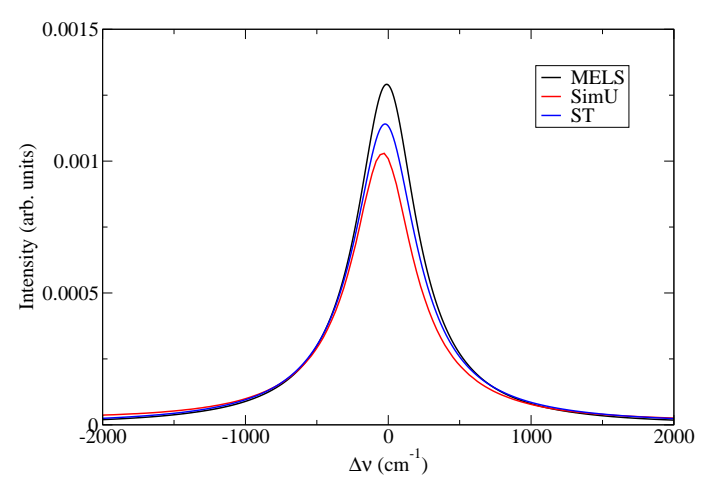

(c)

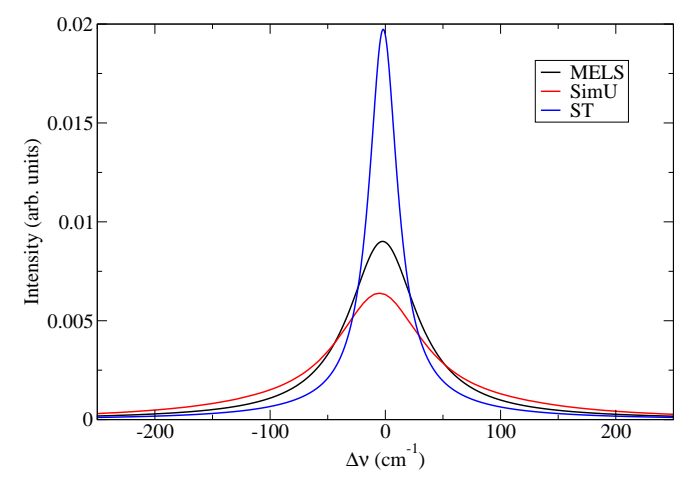

(b)

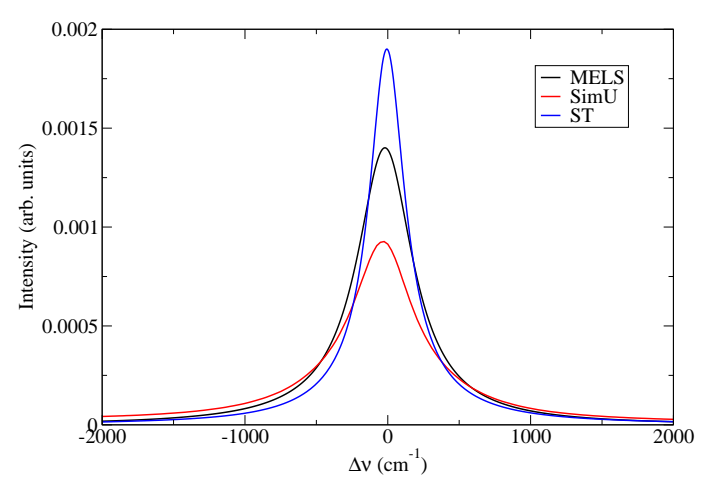

(d)

Figure 6. Sub-cases with quenching. Plasma conditions assumed are as follows: (a) $n_{e}=10^{18} \mathrm{~cm}^{-3}$ and $T=4 \mathrm{eV} ;(\mathbf{b}) n_{e}=10^{18} \mathrm{~cm}^{-3}$ and $T=10 \mathrm{eV} ;(\mathbf{c}) n_{e}=10^{19} \mathrm{~cm}^{-3}$ and $T=4 \mathrm{eV} ;(\mathbf{d}) n_{e}=10^{19} \mathrm{~cm}^{-3}$ and $T=10 \mathrm{eV}$. 


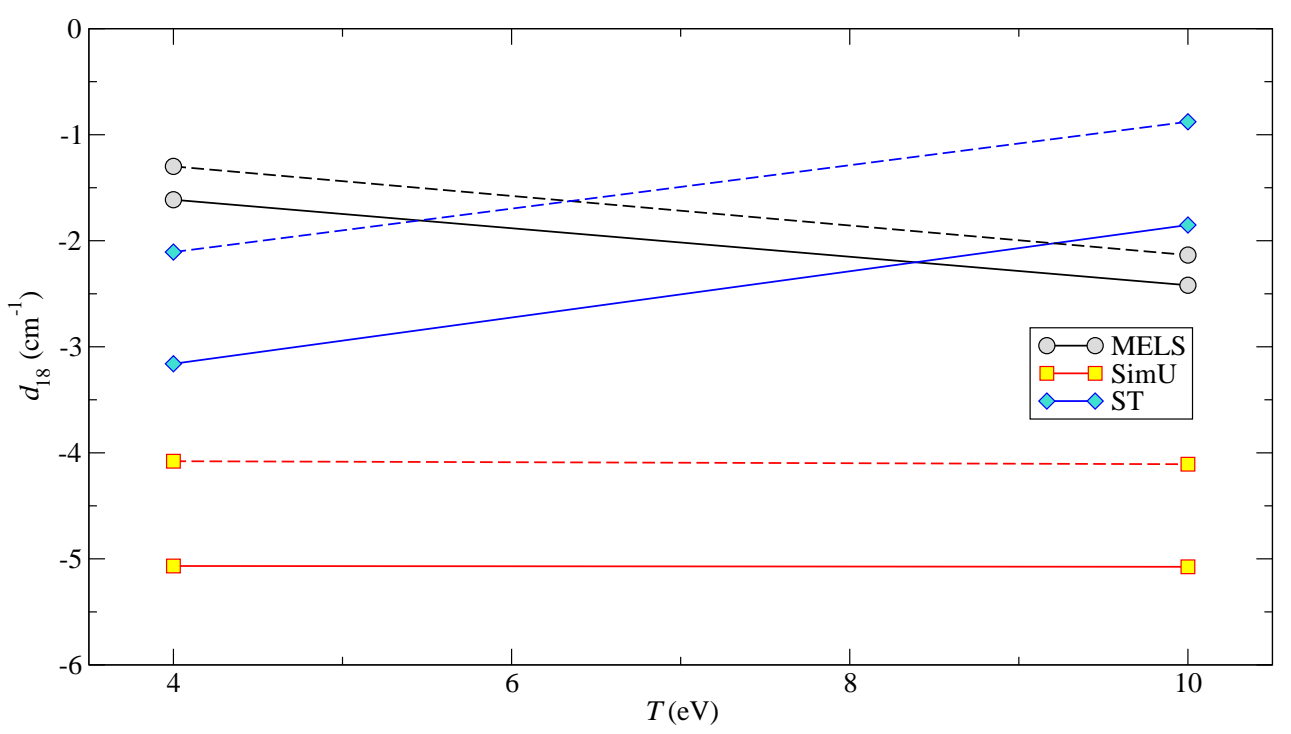

Figure 7. Calculated Stark shift per electron density of $10^{18} \mathrm{~cm}^{-3}$ as a function of plasma temperature. Solid lines: $n_{e}=10^{18} \mathrm{~cm}^{-3}$; dashed lines: $n_{e}=10^{19} \mathrm{~cm}^{-3}$.

\subsection{Experimental Stark Shift and Simulation Results}

Figure 8 shows the present experimental data along with the simulations results. To eliminate the error due to the $n_{e} \neq 0$ reference plasma, we present relative shifts and widths. To achieve a nearly linear relation between the Stark width and the electron density, $w^{3 / 2}$ is used instead of $w$. The sources of the calculation uncertainties are discussed in Section 4. The disagreement between the measurement and the simulation is obvious. All experimental points lie below the best fit to the SimU results and in many cases the latter passes outside the experimental error bars.

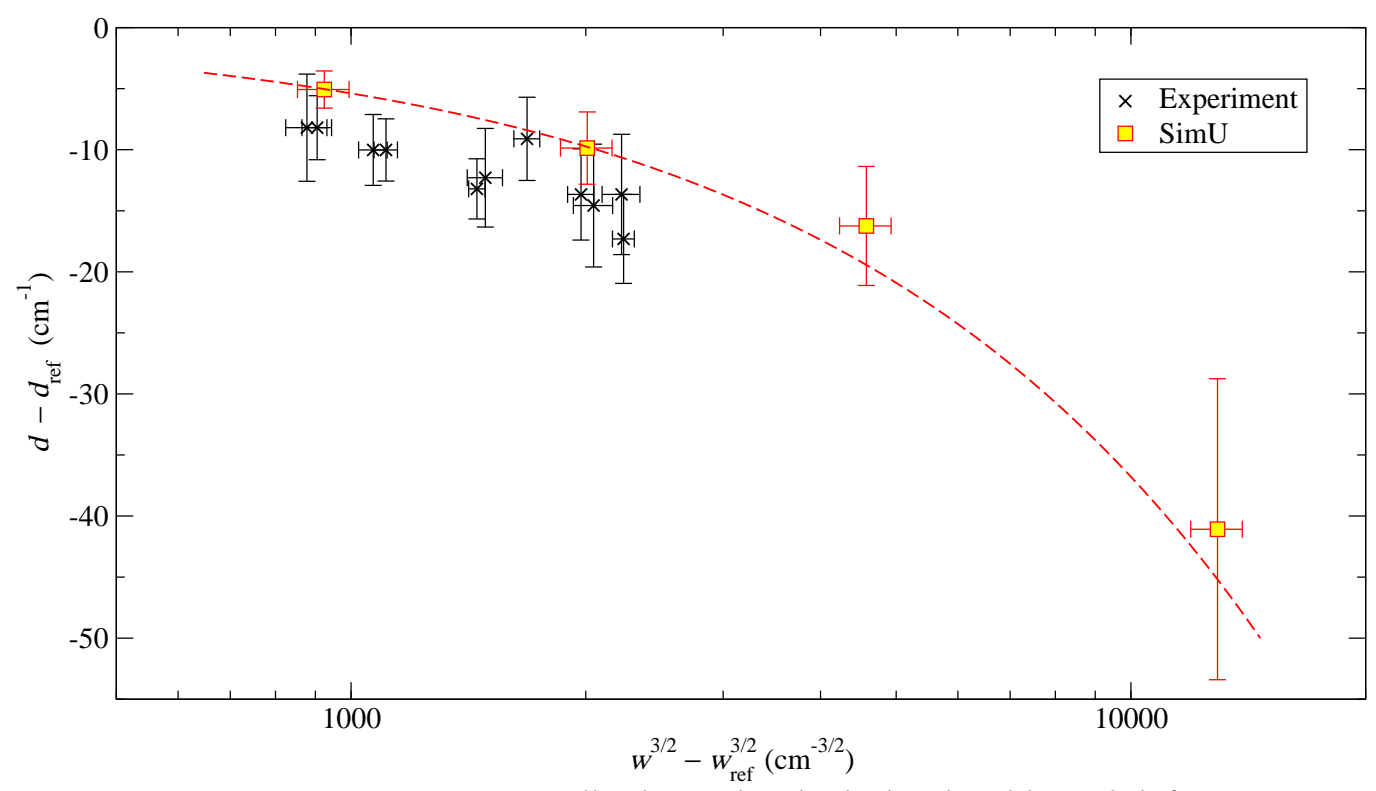

Figure 8. Experimentally obtained and calculated widths and shifts.

\section{Discussion}

Quenching is a pre-requisite for determining the Stark shift of hydrogen-like lines; without a mixing of states with different $n$ values, the Stark shift of a hydrogen-like transition in the dipole 
approximation is zero [11] (however, see a discussion on the detailed balance considerations below). The influence of different states on the line width and shift as calculated by SimU is shown in Figure 9. The higher levels mainly add to the linear term of the Stark width, which is associated with the electron impact contribution. Levels with $n>6$ have practically no additional effect on the width. Accounting only for mixing between the initial and final levels of the line, $n=3-4$ results in a positive ("blue") shift. The influence of $n=5$ reverses the shift, and the even higher $n=6$ makes the shift even larger by absolute value. However, $n=7$ appears to have an almost negligible effect, so higher levels are not taken into account here.
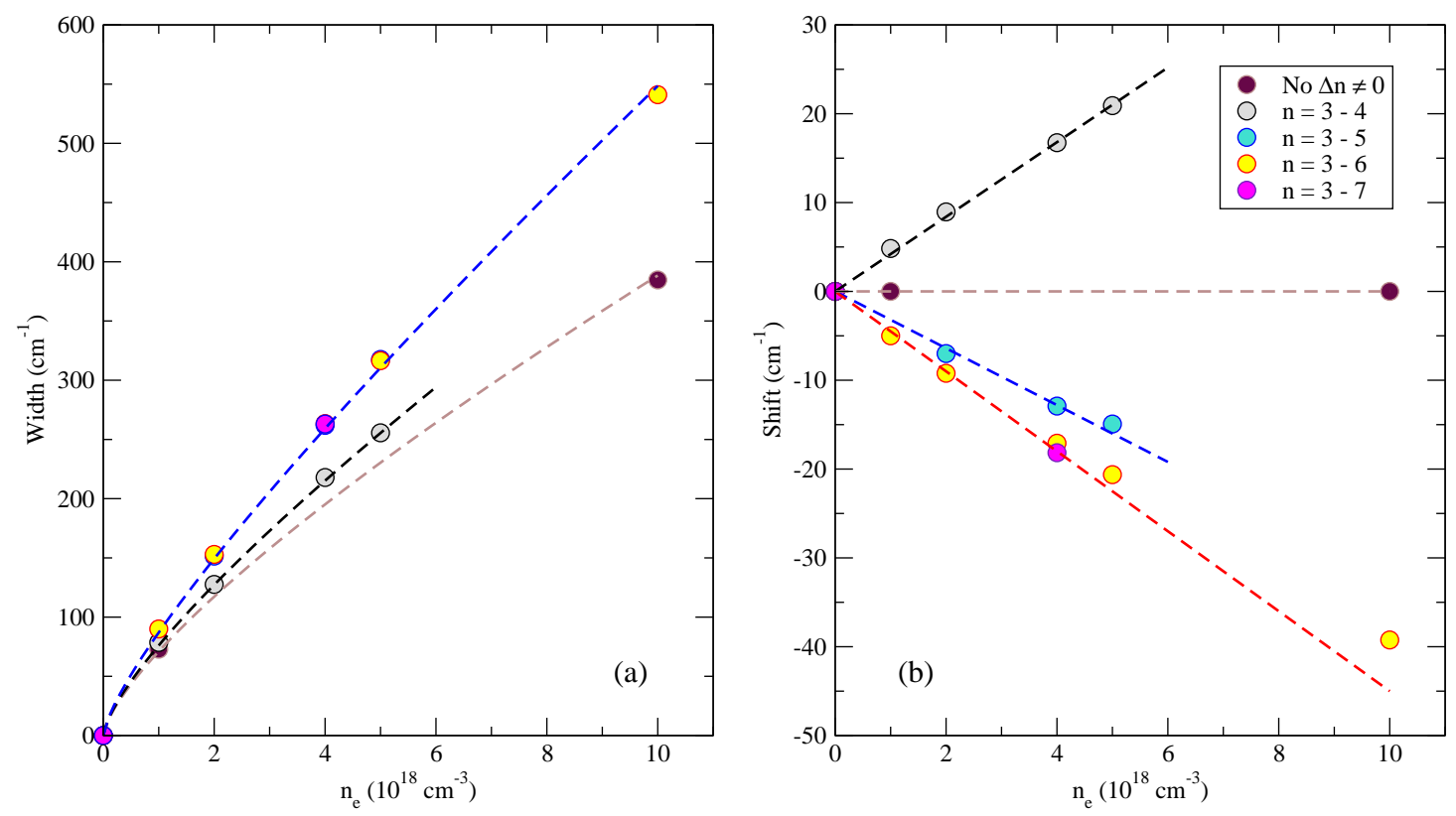

Figure 9. The influence of quenching on the Stark width (a) and shift (b), as calculated by SimU, assuming $T=4 \mathrm{eV}$.

Other physical phenomena not taken into account in either of the calculations here described are quadrupole [31] (and, in principle, higher multipole) terms of the electric-field perturbation and penetrating collisions [32]. Estimates of both effects suggest these phenomena are insufficient to explain the difference between experimental and calculated shifts, although explicit inclusion of both effects in the calculations is certainly desired. The omission of these effects (as well as the states with $n$ values higher than 7 ) contribute to the uncertainties of SimU results, as do the statistical convergence issues [33] and finiteness of the spectral grid. The total estimated uncertainties are designated by the error bars in Figure 8 .

It can be argued that the absence of shifts in the dipole and the no-quenching approximation are due to the classical treatment of plasma electrons, which is the case for all semiclassical models as well as present-day simulations [34]. Considering the plasma bath electrons quantum-mechanically, together with the detailed balance arguments, brings some otherwise unexpected results, e.g., different emission and absorption widths of isolated lines in a non-equilibrium (albeit stationary) plasma [35]. For the same reason, the (frequency-dependent) collision operator is asymmetric. Even though the asymmetry is most noticeable at large detunings $(|\Delta \omega| \sim k T)$, one may argue, invoking a Kramers-Kronig relation between the real and imaginary parts of the collision operator (i.e., electron shift and width), that this asymmetry should result in a non-zero shift.

Due to the time- and space-integrated character of the experimental data presented here, yielding a shifted lineshape together with its low-density "reference" counterpart, systematic errors in the determination of the zero position, the dispersion, or the consideration of spectrometer aberrations, can be excluded. Additionally, very good agreement with experimental line widths and shifts from Büscher et al. [6] contribute to the reliability of the present experimental data. On the other hand, 
there are also contradicting experimental data in the literature, i.e., by Kobilarov et al. [4], which report on significantly lower Stark shifts (which happen to be rather close to the SimU results) measured in a similar plasma configuration.

\section{Conclusions}

We presented experimental Stark widths and shifts of the He II P $\alpha$ line and compared them to a few cases in the literature and to the results of different spectral lineshape codes. A comparison of the calculated lineshapes from the four models and three simulations on pre-defined model cases reveals good agreement of the results for $\Delta n=0$, especially between the simulations. When accounting for quenching, the calculated lineshapes are more controversial. An analysis of the importance of the high- $n$ states suggests that $n$ levels of at least 6 have to be taken into account to obtain an accurate Stark shift. The calculated Stark shift over width, however, is significantly lower than measured in the present experiment. This suggests that there is an additional phenomenon (or phenomena) that influences the Stark shift of He II P $\alpha$ that has yet to be accounted for. Further experiments and development of lineshape calculation codes will hopefully resolve this discrepancy and avail the Stark shift of $\mathrm{He}$ II P $\alpha$ as an accurate indicator of the electron density in dense and possibly optically thick plasmas.

Author Contributions: Spectroscopic measurement and raw data analysis-C.S.; lineshape calculations and comparison with experimental data-all other authors; manuscript writing-C.S. and E.S., with additional contributions from all other authors.

Acknowledgments: Christine Stollberg thanks E. Kroupp and P. Meiri for their help in the experimental arrangement, and D. Mikitchuk for his support during the measurements. The organizational and financial support from the International Atomic Energy Agency for conducting the SLSP workshops is highly appreciated. The work of Christine Stollberg and Evgeny Stambulchik was supported in part by the Israel Science foundation, the DOE-Cornell University Excellence Center (USA), and the Office of Naval Research (USA). The work of Marco A. Gigosos and Diego Gonzalez Herrero was supported by Ministerio de Economia y Competitividad (Spain), project ENE2015-67561-R. The work by Carlos A. Iglesias was performed under the auspices of the U.S. Department of Energy by Lawrence Livermore National Laboratory under Contract DE-AC52-07NA27344.

Conflicts of Interest: The authors declare no conflict of interest.

\section{References}

1. Griem, H.R. Spectral Line Broadening by Plasmas; Academic Press: New York, NY, USA, 1974.

2. Fleurier, C.; Gall, P.L. Shift of the He II P $\alpha$ line in high density plasmas. J. Phys. B At. Mol. Opt. Phys. 1984, 17, 4311-4322. [CrossRef]

3. Pittman, T.L.; Fleurier, C. Plasma shifts of the $\mathrm{He} \mathrm{II}_{\alpha}$ and $\mathrm{P}_{\alpha}$ lines. Phys. Rev. A 1986, 33, 1291-1296. [CrossRef]

4. Kobilarov, R.; Popović, M.V.; Konjević, N. Plasma shift of the He II P $\mathrm{P}_{\alpha}$ line. Phys. Rev. A 1988, 37, 1021-1024. [CrossRef]

5. Gawron, A.; Hey, J.D.; Xu, X.J.; Kunze, H.J. Stark shift of the He II $\mathrm{P}_{\alpha}$ line in a dense plasma. Phys. Rev. A 1989, 40, 7150-7157. [CrossRef]

6. Büscher, S.; Glenzer, S.; Wrubel, T.; Kunze, H.J. Investigation of the He II $\mathrm{P}_{\alpha}$ and He II $\mathrm{P}_{\beta}$ transitions at high densities. J. Phys. B At. Mol. Opt. Phys. 1996, 29, 4107-4125. [CrossRef]

7. Peláez, R.J.; Pérez, C.; González, V.R.; Rodríguez, F.; Aparicio, J.A.; Mar, S. Experimental measurements of shifts and asymmetries of He II $\mathrm{P} \alpha$ and $\mathrm{P} \beta$ spectral lines. J. Phys. B At. Mol. Opt. Phys. 2005, 38, 2505-2517. [CrossRef]

8. Stambulchik, E.; Maron, Y. A study of ion-dynamics and correlation effects for spectral line broadening in plasma: K-shell lines. J. Quant. Spectr. Radiat. Transf. 2006, 99, 730-749. [CrossRef]

9. Rosato, J. Report on the third SLSP code comparison workshop. High Energy Density Phys. 2017, $22,60-63$. [CrossRef]

10. Gigosos, M.A. Stark broadening models for plasma diagnostics. J. Phys. D Appl. Phys. 2014, 47, 343001. [CrossRef]

11. Alexiou, S. On the pure dipole shift for hydrogen lines in a plasma. J. Quant. Spectr. Radiat. Transf. 2003, 81, 13-17. [CrossRef] 
12. Ferri, S.; Calisti, A.; Mossé, C.; Rosato, J.; Talin, B.; Alexiou, S.; Gigosos, M.A.; González, M.Á.; González-Herrero, D.; Lara, N.; et al. Ion dynamics effect on Stark broadened line shapes: A cross comparison of various models. Atoms 2014, 2, 299-318. [CrossRef]

13. Gigosos, M.A.; Cardeñoso, V. New plasma diagnosis tables of hydrogen Stark broadening including ion dynamics. J. Phys. B At. Mol. Opt. Phys. 1996, 29, 4795-4838. [CrossRef]

14. Lara, N. Calculation of Stark Spectra of Strongly Coupled Plasmas Using Molecular Dynamics Simulation. Ph.D. Thesis, Universidad de Valladolid, Valladolid, Spain, 2013.

15. Iglesias, C.A.; Sonnad, V. Robust algorithm for computing quasi-static Stark broadening of spectral lines. High Energy Density Phys. 2010, 6, 399-405. [CrossRef]

16. Calisti, A.; Khelfaoui, F.; Stamm, R.; Talin, B.; Lee, R.W. Model for the line shapes of complex ions in hot and dense plasmas. Phys. Rev. A 1990, 42, 5433-5440. [CrossRef] [PubMed]

17. Stambulchik, E.; Maron, Y. Quasicontiguous frequency-fluctuation model for calculation of hydrogen and hydrogenlike Stark-broadened line shapes in plasmas. Phys. Rev. E 2013, 87, 053108. [CrossRef] [PubMed]

18. Duan, B.; Bari, M.A.; Wu, Z.Q.; Yan, J.; Wang, J.G. Stark-broadened profiles of the Lyman spectral lines in He II ions. J. Phys. B At. Mol. Opt. Phys. 2015, 48, 105701. [CrossRef]

19. Gigosos, M.A.; Fraile, J.; Torres, F. Hydrogen Stark profiles: A simulation-oriented mathematical simplification. Phys. Rev. A 1985, 31, 3509-3511. [CrossRef]

20. Tighe, R.J.; Hooper, C.F. Low-frequency electric microfield distributions in a plasma containing multiplycharged ions: Extended calculations. Phys. Rev. A 1977, 15, 1773-1779. [CrossRef]

21. Dyall, K.G.; Grant, I.P.; Johnson, C.T.; Parpia, F.A.; Plummer, E.P. GRASP: A general-purpose relativistic atomic structure program. Comput. Phys. Commun. 1989, 55, 425-456. [CrossRef]

22. Iglesias, C.; Rogers, F.; Shepherd, R.; Bar-Shalom, A.; Murillo, M.; Kilcrease, D.; Calisti, A.; Lee, R. Fast electric microfield distribution calculations in extreme matter conditions. J. Quant. Spectr. Radiat. Transf. 2000, 65, 303-315. [CrossRef]

23. O'Brien, J.T.; Hooper, C.F. A relaxation theory of plasma-broadened He II lines. J. Quant. Spectr. Radiat. Transf. 1974, 14, 479-496. [CrossRef]

24. Boercker, D.B.; Iglesias, C.A.; Dufty, J.W. Radiative and transport properties of ions in strongly coupled plasmas. Phys. Rev. A 1987, 36, 2254-2264. [CrossRef]

25. Calisti, A.; Mossé, C.; Ferri, S.; Talin, B.; Rosmej, F.; Bureyeva, L.A.; Lisitsa, V.S. Dynamic Stark broadening as the Dicke narrowing effect. Phys. Rev. E 2010, 81, 016406. [CrossRef] [PubMed]

26. Stambulchik, E.; Maron, Y. Stark effect of high- $n$ hydrogen-like transitions: Quasi-contiguous approximation. J. Phys. B At. Mol. Opt. Phys. 2008, 41, 095703. [CrossRef]

27. Pfennig, H.; Trefftz, E. Die Druckverbreiterung der diffusen Heliumlinien, Vergleich zwischen Messung und Theorie im quasistatischen Bereich. Z. Naturforsch. A 1966, 21, 697-718. [CrossRef]

28. Iglesias, C.A. Comparison of electron width models for fast line profile calculations. High Energy Density Phys. 2016, 18, 14-19. [CrossRef]

29. Ferri, S.; Calisti, A.; Stamm, R.; Talin, B.; Lee, R.W.; Klein, L. Electronic broadening model for high- $n$ Balmer line profiles. Phys. Rev. E 1998, 58, R6943-R6946. [CrossRef]

30. Stambulchik, E.; Demura, A.V. Dynamic Stark broadening of Lyman- $\alpha$. J. Phys. B At. Mol. Opt. Phys. 2016, 49, 035701. [CrossRef]

31. Demura, A.V. Beyond the linear Stark effect: A retrospective. Atoms 2018, Submitted.

32. Alexiou, S.; Poquérusse, A. Standard line broadening impact theory for hydrogen including penetrating collisions. Phys. Rev. E 2005, 72, 046404. [CrossRef] [PubMed]

33. Stambulchik, E.; Alexiou, S.; Griem, H.R.; Kepple, P.C. Stark broadening of high principal quantum number hydrogen Balmer lines in low-density laboratory plasmas. Phys. Rev. E 2007, 75, 016401. [CrossRef] [PubMed]

34. Stambulchik, E.; Maron, Y. Plasma line broadening and computer simulations: A mini-review. High Energy Density Phys. 2010, 6, 9-14. [CrossRef]

35. Iglesias, C.A. Electron broadening of isolated lines with stationary non-equilibrium level populations. High Energy Density Phys. 2005, 1, 42-51. [CrossRef]

(C) 2018 by the authors. Licensee MDPI, Basel, Switzerland. This article is an open access article distributed under the terms and conditions of the Creative Commons Attribution (CC BY) license (http:/ / creativecommons.org/licenses/by/4.0/). 